\title{
On Sequence Prediction for Arbitrary Measures
}

\author{
Daniil Ryabko and Marcus Hutter \\ IDSIA, Galleria 2, CH-6928 Manno-Lugano, Switzerland* \\ \{daniil,marcus\}@idsia.ch, http://www.idsia.ch/ \{daniil,marcus\}
}

16 June 2006

\begin{abstract}
Suppose we are given two probability measures on the set of one-way infinite finite-alphabet sequences and consider the question when one of the measures predicts the other, that is, when conditional probabilities converge (in a certain sense) when one of the measures is chosen to generate the sequence. This question may be considered a refinement of the problem of sequence prediction in its most general formulation: for a given class of probability measures, does there exist a measure which predicts all of the measures in the class? To address this problem, we find some conditions on local absolute continuity which are sufficient for prediction and which generalize several different notions which are known to be sufficient for prediction. We also formulate some open questions to outline a direction for finding the conditions on classes of measures for which prediction is possible.
\end{abstract}

\section{Contents}

1 Introduction 2

\begin{tabular}{|lll}
\hline 2 & Notation and Definitions & 5
\end{tabular}

\begin{tabular}{|lll}
\hline 3 & Dominance with Decreasing Coefficients & 7
\end{tabular}

4 Preservation of the Predictive Ability under Summation with $\begin{array}{ll}\text { an Arbitrary Measure } & 11\end{array}$

\begin{tabular}{lll}
\hline 5 & Miscellaneous & 13
\end{tabular}

$\begin{array}{llr}6 & \text { Outlook and Conclusion } & 15\end{array}$

Keywords

Sequence prediction, local absolute continuity, non-stationary measures, average/expected criteria, absolute/KL divergence, mixtures of measures.

*This work was supported by the Swiss NSF grant 200020-107616. 


\section{Introduction}

Let a sequence $x_{t}, t \in \mathbb{N}$ of letters from some finite alphabet $\mathcal{X}$ be generated by some probability measure $\mu$. Having observed the first $n$ letters $x_{1}, \ldots, x_{n}$ we want to predict what is the probability of the next letter being $x$, for each $x \in \mathcal{X}$. This task is motivated by numerous applications - from weather forecasting and stock market prediction to data compression.

If the measure $\mu$ is known completely then the best forecasts one can make for the $(n+1)$ st outcome of a sequence $x_{1}, \ldots, x_{n}$ is $\mu$-conditional probabilities of $x \in \mathcal{X}$ given $x_{1}, \ldots, x_{n}$. On the other hand, it is immediately apparent that if nothing is known about the distribution $\mu$ generating the sequence then no prediction is possible, since for any predictor there is a measure on which it errs (gives inadequate probability forecasts) on every step. Thus one has to restrict the attention to some class of measures. Laplace was perhaps the first to address the question of sequence prediction, his motivation being as follows: Suppose that we know that the Sun has risen every day for 5000 years, what is the probability that it will rise tomorrow? He suggested to assume that the probability that the Sun rises is the same every day and the trials are independent of each other. Thus Laplace considered the task of sequence prediction when the true generating measure belongs to the family of Bernoulli i.i.d. measures with binary alphabet $\mathcal{X}=\{0,1\}$. The predicting measure suggested by Laplace was $\rho_{L}\left(x_{n+1}=1 \mid x_{1}, \ldots, x_{n}\right)=\frac{k+1}{n+2}$ where $k$ is the number of $1 \mathrm{~s}$ in $x_{1}, \ldots, x_{n}$. The conditional probabilities of Laplace's measure $\rho_{L}$ converge to the true conditional probabilities $\mu$-almost surely under any Bernoulli i.i.d measure $\mu$. This approach generalizes to the problem of predicting any finite-memory (e.g. Markovian) measure. Moreover, in Rya88 a measure $\rho_{R}$ was constructed for predicting an arbitrary stationary measure. The conditional probabilities of $\rho_{R}$ converge to the true ones on average, where average is taken over time steps (that is, in Cesaro sense), $\mu$-almost surely for any stationary measure $\mu$. However, as it was shown in the same work, there is no measure for which conditional probabilities converge to the true ones $\mu$-a.s. for every stationary $\mu$. Thus we can see that already for the problem of predicting outcomes of a stationary measure two criteria of prediction arise: prediction in the average (or in Cesaro sense) and prediction on each step, and the solution exists only for the former problem.

But what if the measure generating the sequence is not stationary? A different assumption one can make is that the measure $\mu$ generating the sequence is computable. Solomonoff [Sol64, Eq.(13)] suggested a measure $\xi$ for predicting any computable probability measure. The key observation here is that the class of all computable probability measures is countable; let us denote it by $\left(\nu_{i}\right)_{i \in \mathbb{N}}$. A Bayesian predictor $\xi$ for a countable class of measures $\left(\nu_{i}\right)_{i \in \mathbb{N}}$ is constructed as follows: $\xi(A)=\sum_{i=1}^{\infty} w_{i} \nu_{i}(A)$ for any measurable set $\mathrm{A}$, where the weights $w_{i}$ are positive and sum to on ${ }^{1}$. The best predictor for a measure $\mu$ is the measure $\mu$

\footnotetext{
${ }^{1}$ It is not necessary for prediction that the weights sum to one. In Sol78] and [ZL70] $w_{i}=2^{-K(i)}$ where $K$ stands for the prefix Kolmogorov complexity, and so the weights do not sum to 1 . Further,
} 
itself. The Bayesian predictor simply takes the weighted average of the predictors for all measures in the class - for countable classes this is possible. It was shown by Solomonoff [Sol78] that $\xi$-conditional probabilities converge to $\mu$-conditional probabilities almost surely for any computable measure $\mu$. In fact this is a special case of a more general (though without convergence rate) result of Blackwell and Dubins [BD62] which states that if a measure $\mu$ is absolutely continuous with respect to a measure $\rho$ then $\rho$ converges to $\mu$ in total variation $\mu$-almost surely. Convergence in total variation means prediction in a very strong sense - convergence of conditional probabilities of arbitrary events (not just the next outcome), or prediction with arbitrary fast growing horizon. Since for $\xi$ we have $\xi(A) \geq w_{i} \nu_{i}(A)$ for every measurable set $A$ and for every $\nu_{i}$, each $\nu_{i}$ is absolutely continuous with respect to $\xi$.

Thus the problem of sequence prediction for certain classes of measures (such as the class of all stationary measures or the class of all computable measures) was often addressed in the literature. Although the mentioned classes of measures are sufficiently interesting, it is often hard to decide in applications with which assumptions does a problem at hand comply; not to mention such practical issues as that a predicting measure for all computable measures is necessarily non-computable itself. Moreover, to be able to generalize the solutions of the sequence prediction problem to such problems as active learning, where outcomes of a sequence may depend on actions of the predictor, one has to understand better under which conditions the problem of sequence prediction is solvable. In particular, in active learning, the stationarity assumption does not seem to be applicable (since the predictions are non-stationary), although, say, the Markov assumption is often applicable and is extensively studied. Thus, we formulate the following general questions which we start to address in the present work:

General motivating questions. For which classes of measures is sequence prediction possible? Under which conditions does a measure $\rho$ predict a measure $\mu$ ?

As we have seen, these questions have many facets, and in particular there are many criteria of prediction to be considered, such as almost sure convergence of conditional probabilities, convergence in average, etc. Extensive as the literature on sequence prediction is, these questions in their full generality have not received much attention. One line of research which exhibits this kind of generality consists in extending the result of Blackwell and Dubins mentioned above, which states that if $\mu$ is absolutely continuous with respect to $\rho$, then $\rho$ predicts $\mu$ in total variation distance. In [JKS99] a question of whether, given a class of measures $\mathcal{C}$ and a prior ("meta"-measure) $\lambda$ over this class of measures, the conditional probabilities of a Bayesian mixture of the class $\mathcal{C}$ w.r.t. $\lambda$ converge to the true $\mu$-probabilities (weakly merge, in terminology of [JKS99]) for $\lambda$-almost any measure $\mu$ in $\mathcal{C}$. This question can be considered solved, since the authors provide necessary and sufficient conditions on the measure given by the mixture of the class $\mathcal{C}$ w.r.t. $\lambda$ under which

the $\nu$ and $\xi$ are only semi-measures. 
prediction is possible. The major difference from the general questions we posed above is that we do not wish to assume that we have a measure on our class of measures. For large (non-parametric) classes of measures it may not be intuitive which measure over it is natural; rather, the question is whether a "natural" measure which can be used for prediction exists.

To address the general questions posed, we start with the following observation. As it was mentioned, for a Bayesian mixture $\xi$ of a countable class of measures $\nu_{i}$, $i \in \mathbb{N}$, we have $\xi(A) \geq w_{i} \nu_{i}(A)$ for any $i$ and any measurable set $A$, where $w_{i}$ is a constant. This condition is stronger than the assumption of absolute continuity and is sufficient for prediction in a very strong sense. Since we are willing to be satisfied with prediction in a weaker sense (e.g. convergence of conditional probabilities), let us make a weaker assumption: Say that a measure $\rho$ dominates a measure $\mu$ with coefficients $c_{n}>0$ if

$$
\rho\left(x_{1}, \ldots, x_{n}\right) \geq c_{n} \mu\left(x_{1}, \ldots, x_{n}\right)
$$

for all $x_{1}, \ldots, x_{n}$.

The first concrete question we pose is, under what conditions on $c_{n}$ does (1) imply that $\rho$ predicts $\mu$ ? Observe that if $\rho\left(x_{1}, \ldots, x_{n}\right)>0$ for any $x_{1}, \ldots, x_{n}$ then any measure $\mu$ is locally absolutely continuous with respect to $\rho$ (that is, the measure $\mu$ restricted to the first $n$ trials $\left.\mu\right|_{\mathcal{X}^{n}}$ is absolutely continuous w.r.t. $\left.\rho\right|_{\mathcal{X}^{n}}$ for each $n$ ), and moreover, for any measure $\mu$ some constants $c_{n}$ can be found that satisfy (1). For example, if $\rho$ is Bernoulli i.i.d. measure with parameter $\frac{1}{2}$ and $\mu$ is any other measure, then (1) is (trivially) satisfied with $c_{n}=2^{-n}$. Thus we know that if $c_{n} \equiv c$ then $\rho$ predicts $\mu$ in a very strong sense, whereas exponentially decreasing $c_{n}$ are not enough for prediction. Perhaps somewhat surprisingly, we will show that dominance with any subexponentially decreasing coefficients is sufficient for prediction, in a weak sense of convergence of expected averages. Dominance with any polynomially decreasing coefficients, and also with coefficients decreasing (for example) as $c_{n}=\exp (-\sqrt{n} / \log n)$, is sufficient for (almost sure) prediction on average (i.e. in Cesaro sense). However, for prediction on every step we have a negative result: for any dominance coefficients that go to zero there exists a pair of measures $\rho$ and $\mu$ which satisfy (1) but $\rho$ does not predict $\mu$ in the sense of almost sure convergence of probabilities. Thus the situation is similar to that for predicting any stationary measure: prediction is possible in the average but not on every step.

Note also that for Laplace's measure $\rho_{L}$ it can be shown that $\rho_{L}$ dominates any i.i.d. measure $\mu$ with linearly decreasing coefficients $c_{n}=\frac{1}{n+1}$; a generalization of $\rho_{L}$ for predicting all measures with memory $k$ (for a given $k$ ) dominates them with polynomially decreasing coefficients. Thus dominance with decreasing coefficients generalizes (in a sense) predicting countable classes of measures (where we have dominance with a constant), absolute continuity (via local absolute continuity), and predicting i.i.d. and finite-memory measures.

Another way to look for generalizations is as follows. The Bayes mixture $\xi$, being a sum of countably many measures (predictors), possesses some of their predicting 
properties. In general, which predictive properties are preserved under summation? In particular, if we have two predictors $\rho_{1}$ and $\rho_{2}$ for two classes of measures, we are interested in the question whether $\frac{1}{2}\left(\rho_{1}+\rho_{2}\right)$ is a predictor for the union of the two classes. An answer to this question would improve our understanding of how far a class of measures for which a predicting measure exists can be extended without losing this property.

Thus, the second question we consider is the following: suppose that a measure $\rho$ predicts $\mu$ (in some weak sense), and let $\chi$ be some other measure (e.g. a predictor for a different class of measures). Does the measure $\rho^{\prime}=\frac{1}{2}(\rho+\chi)$ still predict $\mu$ ? That is, we ask to which prediction quality criteria does the idea of taking a Bayesian sum generalize. Absolute continuity is preserved under summation along with it's (strong) prediction ability. It was mentioned in [RA06] that prediction in the (weak) sense of convergence of expected averages of conditional probabilities is preserved under summation. Here we find that several stronger notions of prediction are not preserved under summation.

Thus we address the following two questions. Is dominance with decreasing coefficients sufficient for prediction in some sense, under some conditions on the coefficients? And, if a measure $\rho$ predicts a measure $\mu$ in some sense, does the measure $\frac{1}{2}(\rho+\chi)$ also predict $\mu$ in the same sense, where $\chi$ is an arbitrary measure? Considering different criteria of prediction (a.s. convergence of conditional probabilities, a.s. convergence of averages, etc.) in the above two questions we obtain not two but many different questions, some of which we answer in the positive and some in the negative, yet some are left open.

Contents. The paper is organized as follows. Section 2 introduces necessary notation and measures of divergence of probability measures. Section 3 addresses the question of whether dominance with decreasing coefficients is sufficient for prediction, while in Section 4 we consider the question of summing a predictor with an arbitrary measure. Both sections 3 and 4 also propose some open questions and directions for future research. In Section 5 we discuss some interesting special cases of the questions considered, and also some related problems.

\section{Notation and Definitions}

We consider processes on the set of one-way infinite sequences $\mathcal{X}^{\infty}$ where $\mathcal{X}$ is a finite set (alphabet). In the examples we will often assume $\mathcal{X}=\{0,1\}$. The notation $x_{1: n}$ is used for $x_{1}, \ldots, x_{n}$ and $x_{<n}$ for $x_{1}, \ldots, x_{n-1}, x_{t} \in \mathcal{X}$. The symbol $\mu$ is reserved for the "true" measure generating examples. We use $\mathbf{E}_{\nu}$ for expectation with respect to a measure $\nu$ and simply $\mathbf{E}$ for $\mathbf{E}_{\mu}$ (expectation with respect to the "true" measure generating examples).

For two measures $\mu$ and $\rho$ define the following measures of divergence.

(d) Kullblack-Leibler (KL) divergence 


$$
d_{n}\left(\mu, \rho \mid x_{<n}\right)=\sum_{x \in \mathcal{X}} \mu\left(x_{n}=x \mid x_{<n}\right) \log \frac{\mu\left(x_{n}=x \mid x_{<n}\right)}{\rho\left(x_{n}=x \mid x_{<n}\right)},
$$

$(\bar{d})$ average KL divergence $\bar{d}_{n}\left(\mu, \rho \mid x_{1: n}\right)=\frac{1}{n} \sum_{t=1}^{n} d_{t}\left(\mu, \rho \mid x_{<n}\right)$,

(a) absolute distance $a_{n}\left(\mu, \rho \mid x_{<n}\right)=\sum_{x \in \mathcal{X}}\left|\mu\left(x_{n}=x \mid x_{<n}\right)-\rho\left(x_{n}=x \mid x_{<n}\right)\right|$,

$(\bar{a})$ average absolute distance $\bar{a}_{n}\left(\mu, \rho \mid x_{1: n}\right)=\frac{1}{n} \sum_{t=1}^{n} a_{t}\left(\mu, \rho \mid x_{<n}\right)$.

\section{Definition 1 (Convergence concepts) We say that $\rho$ predicts $\mu$}

(d) in $K L$ divergence if $d_{n}\left(\mu, \rho \mid x_{<n}\right) \rightarrow 0 \mu$-a.s.,

$(\bar{d})$ in average $K L$ divergence if $\bar{d}_{n}\left(\mu, \rho \mid x_{1: n}\right) \rightarrow 0 \mu$-a.s.

$(\mathbf{E} \bar{d})$ in expected average $K L$ divergence if $\mathbf{E}_{\mu} \bar{d}_{n}\left(\mu, \rho \mid x_{1: n}\right) \rightarrow 0$,

(a) in absolute distance if $a_{n}\left(\mu, \rho \mid x_{<n}\right) \rightarrow 0 \mu$-a.s.,

$(\bar{a})$ in average absolute distance if $\bar{a}_{n}\left(\mu, \rho \mid x_{1: n}\right) \rightarrow 0 \mu$-a.s.

$(\mathbf{E} \bar{a})$ in expected average absolute distance if $\mathbf{E}_{\mu} \bar{a}_{n}\left(\mu, \rho \mid x_{1: n}\right) \rightarrow 0$.

The argument $x_{1: n}$ will be often left implicit in our notation. A measure $\rho$ converges to $\mu$ in total variation $(t v)$ if $\sup _{A \subset \sigma\left(\bigcup_{t=n}^{\infty} \mathcal{X}^{t}\right)}\left|\mu\left(A \mid x_{<n}\right)-\rho\left(A \mid x_{<n}\right)\right| \rightarrow 0 \mu-$ almost surely. Some other measures of prediction ability are considered in Section 5 . The following implications hold (and are complete and strict):

$$
\begin{aligned}
& d \Rightarrow \bar{d} \quad \mathbf{E} \bar{d} \\
& t v \Rightarrow \begin{array}{l}
\Downarrow \\
\Downarrow
\end{array} \underset{\bar{a}}{\Downarrow} \begin{array}{c}
\Downarrow \\
\mathbf{E} \bar{a}
\end{array}
\end{aligned}
$$

to be understood as e.g.: if $\bar{d}_{n} \rightarrow 0$ a.s. then $\bar{a}_{n} \rightarrow 0$ a.s, or, if $\mathbf{E} \bar{d}_{n} \rightarrow 0$ then $\mathbf{E} \bar{a}_{n} \rightarrow 0$. The horizontal implications $\Rightarrow$ follow immediately from the definitions, and the $\Downarrow$ follow from the following Lemma:

Lemma $2\left(\boldsymbol{a}^{\mathbf{2}} \leq \mathbf{2 d}\right)$ For all measures $\rho$ and $\mu$ and sequences $x_{1: \infty}$ we have: $a_{t}^{2} \leq 2 d_{t}$ and $\bar{a}_{n}^{2} \leq 2 \bar{d}_{n}$ and $\left(\mathbf{E} \bar{a}_{n}\right)^{2} \leq 2 \mathbf{E} \bar{d}_{n}$.

Proof. Pinsker's inequality [Hut05, Lem.3.11a] implies $a_{t}^{2} \leq 2 d_{t}$. Using this and Jensen's inequality for the average $\frac{1}{n} \sum_{t=1}^{n}[\ldots]$ we get

$$
2 \bar{d}_{n}=\frac{1}{n} \sum_{t=1}^{n} 2 d_{t} \geq \frac{1}{n} \sum_{t=1}^{n} a_{t}^{2} \geq\left(\frac{1}{n} \sum_{t=1}^{n} a_{t}\right)^{2}=\bar{a}_{n}^{2}
$$

Using this and Jensen's inequality for the expectation $\mathbf{E}$ we get $2 \mathbf{E} \bar{d}_{n} \geq \mathbf{E} \bar{a}_{n}^{2} \geq\left(\mathbf{E} \bar{a}_{n}\right)^{2}$. 


\section{Dominance with Decreasing Coefficients}

First we consider the question whether property (1) is sufficient for prediction.

Definition 3 (Dominance) We say that a measure $\rho$ dominates a measure $\mu$ with coefficients $c_{n}>0$ iff

$$
\rho\left(x_{1: n}\right) \geq c_{n} \mu\left(x_{1: n}\right) .
$$

Suppose that $\rho$ dominates $\mu$ with decreasing coefficients $c_{n}$. Does $\rho$ predict $\mu$ in (expected, expected average) KL divergence (absolute distance)? First let us give an example.

Proposition 4 (Dominance of Laplace's measure) Let $\rho_{L}$ be the Laplace measure, given by $\rho_{L}\left(x_{n+1}=a \mid x_{1: n}\right)=\frac{k+1}{n+|\mathcal{X}|}$ for any $a \in \mathcal{X}$ and any $x_{1: n} \in \mathcal{X}^{n}$, where $k$ is the number of occurrences of a in $x_{1: n}$. Then

$$
\rho_{L}\left(x_{1: n}\right) \geq \frac{n !}{(n+|\mathcal{X}|-1) !} \mu\left(x_{1: n}\right)
$$

for any measure $\mu$ which generates independently and identically distributed symbols. This bound is sharp.

Proof. We will only give the proof for $\mathcal{X}=\{0,1\}$, the general case is analogous. To calculate $\rho_{L}\left(x_{1: n}\right)$ observe that it only depends on the number of $0 \mathrm{~s}$ and $1 \mathrm{~s}$ in $x_{1: n}$ and not on their order. Thus we compute $\rho_{L}\left(x_{1: n}\right)=\frac{k !(n-k) !}{(n+1) !}$ where $k$ is the number of 1 s. For any measure $\mu$ such that $\mu\left(x_{n}=1\right)=p$ for some $p \in[0,1]$ independently for all $n$, and for Laplace measure $\rho_{L}$ we have

$$
\begin{aligned}
\frac{\mu\left(x_{1: n}\right)}{\rho_{L}\left(x_{1: n}\right)} & =\frac{(n+1) !}{k !(n-k) !} p^{k}(1-p)^{n-k} \\
& =(n+1)\left(\begin{array}{l}
n \\
k
\end{array}\right) p^{k}(1-p)^{n-k} \\
& \leq(n+1) \sum_{k=1}^{n}\left(\begin{array}{l}
n \\
k
\end{array}\right) p^{k}(1-p)^{n-k}=n+1
\end{aligned}
$$

for any $n$-letter word $x_{1}, \ldots, x_{n}$ where $k$ is the number of $1 \mathrm{~s}$ in it. The bound is attained when $p=1$, so that $k=n, \mu\left(x_{1: n}\right)=1$, and $\rho_{L}\left(x_{1: n}\right)=\frac{1}{n+1}$.

Thus for Laplace's measure $\rho_{L}$ and binary $\mathcal{X}$ we have $c_{n}=\mathcal{O}\left(\frac{1}{n}\right)$. As it was mentioned in the introduction, in general, exponentially decreasing coefficients $c_{n}$ are not sufficient for prediction, since (1) is satisfied with $\rho$ being a Bernoulli i.i.d. measure and $\mu$ any other measure. On the other hand, the following proposition shows that in a weak sense of convergence in expected average KL divergence (or absolute distance) the property (1) with subexponentially decreasing $c_{n}$ is sufficient. We also remind that if $c_{n}$ are bounded from below then prediction in the strong sense of total variation is possible. 
Theorem $5(\mathbf{E} \overline{\boldsymbol{d}} \rightarrow \mathbf{0}$ and $\mathbf{E} \overline{\boldsymbol{a}} \longrightarrow \mathbf{0})$ Let $\mu$ and $\rho$ be two measures on $\mathcal{X}^{\infty}$ and suppose that $\rho\left(x_{1: n}\right) \geq c_{n} \mu\left(x_{1: n}\right)$ for any $x_{1: n}$, where $c_{n}$ are positive constants satisfying $\frac{1}{n} \log c_{n}^{-1} \rightarrow 0$. Then $\rho$ predicts $\mu$ in expected average $K L$ divergence $\mathbf{E}_{\mu} \bar{d}_{n}(\mu, \rho) \rightarrow 0$ and in expected average absolute distance $\mathbf{E}_{\mu} \bar{a}_{n}(\mu, \rho) \rightarrow 0$.

The proof of this proposition is based on the same idea as the proof of convergence of Solomonoff predictor to any of its summands in Rya88, see also [Hut05].

Proof. For convergence in average expected KL divergence we have

$$
\begin{aligned}
\mathbf{E}_{\mu} \bar{d}_{n}(\mu, \rho) & =\frac{1}{n} \mathbf{E} \sum_{t=1}^{n} \sum_{x_{t} \in \mathcal{X}} \mu\left(x_{t} \mid x_{<t}\right) \log \frac{\mu\left(x_{t} \mid x_{<t}\right)}{\rho\left(x_{t} \mid x_{<t}\right)}=\frac{1}{n} \sum_{t=1}^{n} \mathbf{E E}^{t} \log \frac{\mu\left(x_{t} \mid x_{<t}\right)}{\rho\left(x_{t} \mid x_{<t}\right)} \\
& =\frac{1}{n} \mathbf{E} \log \prod_{t=1}^{n} \frac{\mu\left(x_{t} \mid x_{<t}\right)}{\rho\left(x_{t} \mid x_{<t}\right)}=\frac{1}{n} \mathbf{E} \log \frac{\mu\left(x_{1: n}\right)}{\rho\left(x_{1: n}\right)} \leq \frac{1}{n} \log c_{n}^{-1} \rightarrow 0,
\end{aligned}
$$

where $\mathbf{E}^{t}$ stands for the $\mu$-expectation over $x_{t}$ conditional on $x_{<t}$.

The statement for expected average distance follows from this and Lemma 2 .

With a stronger condition on $c_{n}$ prediction in average KL divergence can be established.

Theorem $6(\overline{\boldsymbol{d}} \rightarrow \mathbf{0}$ and $\overline{\boldsymbol{a}} \rightarrow \mathbf{0})$ Let $\mu$ and $\rho$ be two measures on $\mathcal{X}^{\infty}$ and suppose that $\rho\left(x_{1: n}\right) \geq c_{n} \mu\left(x_{1: n}\right)$ for every $x_{1: n}$, where $c_{n}$ are positive constants satisfying

$$
\sum_{n=1}^{\infty} \frac{\left(\log c_{n}^{-1}\right)^{2}}{n^{2}}<\infty
$$

Then $\rho$ predicts $\mu$ in average $K L$ divergence $\bar{d}_{n}(\mu, \rho) \rightarrow 0 \mu$-a.s. and in average absolute distance $\bar{a}_{n}(\mu, \rho) \rightarrow 0 \mu$-a.s.

In particular, the condition (2) on the coefficients is satisfied for polynomially decreasing coefficients, or for $c_{n}=\exp (-\sqrt{n} / \log n)$.

Proof. Again the second statement (about absolute distance) follows from the first one and Lemma 2, so that we only have to prove the statement about KL divergence.

Introduce the symbol $\mathbf{E}^{n}$ for $\mu$-expectation over $x_{n}$ conditional on $x_{<n}$. Consider random variables $l_{n}=\log \frac{\mu\left(x_{n} \mid x_{<n}\right)}{\rho\left(x_{n} \mid x_{<n}\right)}$ and $\bar{l}_{n}=\frac{1}{n} \sum_{t=1}^{n} l_{t}$. Observe that $d_{n}=\mathbf{E}^{n} l_{n}$, so that the random variables $m_{n}=l_{n}-d_{n}$ form a martingale difference sequence (that is, $\left.\mathbf{E}^{n} m_{n}=0\right)$. Let also $\bar{m}_{n}=\frac{1}{n} \sum_{t=1}^{n} m_{t}$. We will show that $\bar{m}_{n} \rightarrow 0 \mu$-a.s. and $\bar{l}_{n} \rightarrow 0$ $\mu$-a.s. which implies $\bar{d}_{n} \rightarrow 0 \mu$-a.s.

Note that

$$
\bar{l}_{n}=\frac{1}{n} \log \frac{\mu\left(x_{1: n}\right)}{\rho\left(x_{1: n}\right)} \leq \frac{\log c_{n}^{-1}}{n} \rightarrow 0 .
$$

Thus to show that $\bar{l}_{n}$ goes to 0 we need to bound it from below. It is easy to see that $n \bar{l}_{n}$ is $\left(\mu\right.$-a.s.) bounded from below by a constant, since $\frac{\rho\left(x_{1: n}\right)}{\mu\left(x_{1: n}\right)}$ is a $\mu$ martingale whose expectation is 1 , and so it converges to a finite limit $\mu$-a.s. by Doob's submartingale convergence theorem, see e.g. [Shi96, p.508]. 
Next we will show that $\bar{m}_{n} \rightarrow 0 \mu$-a.s. We have

$$
\begin{aligned}
m_{n} & =\log \frac{\mu\left(x_{1: n}\right)}{\rho\left(x_{1: n}\right)}-\log \frac{\mu\left(x_{<n}\right)}{\rho\left(x_{<n}\right)}-\mathbf{E}^{n} \log \frac{\mu\left(x_{1: n}\right)}{\rho\left(x_{1: n}\right)}+\mathbf{E}^{n} \log \frac{\mu\left(x_{<n}\right)}{\rho\left(x_{<n}\right)} \\
& =\log \frac{\mu\left(x_{1: n}\right)}{\rho\left(x_{1: n}\right)}-\mathbf{E}^{n} \log \frac{\mu\left(x_{1: n}\right)}{\rho\left(x_{1: n}\right)} .
\end{aligned}
$$

Let $f(n)$ be some function monotonically increasing to infinity such that

$$
\sum_{n=1}^{\infty} \frac{\left(\log c_{n}^{-1}+f(n)\right)^{2}}{n^{2}}<\infty
$$

(e.g. choose $f(n)=\log n$ and exploit $\left(\log c_{n}^{-1}+f(n)\right)^{2} \leq 2\left(\log c_{n}^{-1}\right)^{2}+2 f(n)^{2}$ and (2).) For a sequence of random variables $\lambda_{n}$ define

$$
\left(\lambda_{n}\right)^{+(f)}= \begin{cases}\lambda_{n} & \text { if } \lambda_{n} \geq-f(n) \\ 0 & \text { otherwise }\end{cases}
$$

and $\lambda_{n}^{-(f)}=\lambda_{n}-\lambda_{n}^{+(f)}$. Introduce also

$$
m_{n}^{+}=\left(\log \frac{\mu\left(x_{1: n}\right)}{\rho\left(x_{1: n}\right)}\right)^{+(f)}-\mathbf{E}^{n}\left(\log \frac{\mu\left(x_{1: n}\right)}{\rho\left(x_{1: n}\right)}\right)^{+(f)}
$$

$m_{n}^{-}=m_{n}-m_{n}^{+}$and the averages $\bar{m}_{n}^{+}$and $\bar{m}_{n}^{-}$. Observe that $m_{n}^{+}$is a martingale difference sequence. Hence to establish the convergence $\bar{m}_{n}^{+} \rightarrow 0$ we can use the martingale strong law of large numbers [Shi96, p.501], which states that, for a martingale difference sequence $\lambda_{n}$, if $\mathbf{E}\left(n \bar{\lambda}_{n}\right)^{2}<\infty$ and $\sum_{n=1}^{\infty} \mathbf{E} \lambda_{n}^{2} / n^{2}<\infty$ then $\bar{\lambda}_{n} \rightarrow 0$ a.s. Indeed, for $m_{n}^{+}$the first condition is trivially satisfied (since the expectation in question is a finite sum of finite numbers), and the second follows from the fact that $\left|m_{n}^{+}\right| \leq \log c_{n}^{-1}+f(n)$ and (3).

Furthermore, we have

$$
m_{n}^{-}=\left(\log \frac{\mu\left(x_{1: n}\right)}{\rho\left(x_{1: n}\right)}\right)^{-(f)}-\mathbf{E}^{n}\left(\log \frac{\mu\left(x_{1: n}\right)}{\rho\left(x_{1: n}\right)}\right)^{-(f)} .
$$

As it was mentioned before, $\log \frac{\mu\left(x_{1: n}\right)}{\rho\left(x_{1: n}\right)}$ converges $\mu$-a.s. either to (positive) infinity or to a finite number. Hence $\left(\log \frac{\mu\left(x_{1: n}\right)}{\rho\left(x_{1: n}\right)}\right)^{-(f)}$ is non-zero only a finite number of times, and so its average goes to zero. To see that $\mathbf{E}^{n}\left(\log \frac{\mu\left(x_{1: n}\right)}{\rho\left(x_{1: n}\right)}\right)^{-(f)} \rightarrow 0$ we write

$$
\begin{aligned}
\mathbf{E}^{n}\left(\log \frac{\mu\left(x_{1: n}\right)}{\rho\left(x_{1: n}\right)}\right)^{-(f)} & =\sum_{x_{n} \in \mathcal{X}} \mu\left(x_{n} \mid x_{<n}\right)\left(\log \frac{\mu\left(x_{<n}\right)}{\rho\left(x_{<n}\right)}+\log \frac{\mu\left(x_{n} \mid x_{<n}\right)}{\rho\left(x_{n} \mid x_{<n}\right)}\right)^{-(f)} \\
& \geq \sum_{x_{n} \in \mathcal{X}} \mu\left(x_{n} \mid x_{<n}\right)\left(\log \frac{\mu\left(x_{<n}\right)}{\rho\left(x_{<n}\right)}+\log \mu\left(x_{n} \mid x_{<n}\right)\right)^{-(f)}
\end{aligned}
$$


and note that the first term in brackets is bounded from below, and so for the sum in brackets to be less than $-f(n)$ (which is unbounded) the second term $\log \mu\left(x_{n} \mid x_{<n}\right)$ has to go to $-\infty$, but then the expectation goes to zero since $\lim _{u \rightarrow 0} u \log u=0$.

Thus we conclude that $\bar{m}_{n}^{-} \rightarrow 0 \mu$-a.s., which together with $\bar{m}_{n}^{+} \rightarrow 0 \mu$-a.s. implies $\bar{m}_{n} \rightarrow 0 \mu$-a.s., which, finally, together with $\bar{l}_{n} \rightarrow 0 \mu$-a.s. implies $\bar{d}_{n} \rightarrow 0 \mu$-a.s.

However, no form of dominance with decreasing coefficients is sufficient for prediction in absolute distance or KL divergence, as the following negative result states.

Proposition $\mathbf{7}(\boldsymbol{d} \nrightarrow \mathbf{0}$ and $\boldsymbol{a} \nrightarrow \mathbf{0})$ For each sequence of positive numbers $c_{n}$ that goes to 0 there exist measures $\mu$ and $\rho$ and a number $\epsilon>0$ such that $\rho\left(x_{1: n}\right) \geq c_{n} \mu\left(x_{1: n}\right)$ for all $x_{1: n}$, yet $a_{n}\left(\mu, \rho \mid x_{1: n}\right)>\epsilon$ and $d_{n}\left(\mu, \rho \mid x_{1: n}\right)>\epsilon$ infinitely often $\mu$-a.s.

Proof. Let $\mu$ be concentrated on the sequence $11111 \ldots$ (that is $\mu\left(x_{n}=1\right)=1$ for all $n$ ), and let $\rho\left(x_{n}=1\right)=1$ for all $n$ except for a subsequence of steps $n=n_{k}$, $k \in \mathbb{N}$ on which $\rho\left(x_{n_{k}}=1\right)=1 / 2$ independently of each other. It is easy to see that choosing $n_{k}$ sparse enough we can make $\rho\left(1_{1} \ldots 1_{n}\right)$ decrease arbitrary slowly; yet $\left|\mu\left(x_{n_{k}}\right)-\rho\left(x_{n_{k}}\right)\right|=1 / 2$ for all $k$.

Thus for the first question — whether dominance with some coefficients decreasing to zero is sufficient for prediction, we have the following table of questions and answers, where, in fact, positive answers for $a_{n}$ are implied by positive answers for $d_{n}$ and vice versa for the negative answers:

\begin{tabular}{|c|c|c|c|c|c|}
\hline $\mathbf{E} \bar{d}_{n}$ & $\bar{d}_{n}$ & $d_{n}$ & $\mathbf{E} \bar{a}_{n}$ & $\bar{a}_{n}$ & $a_{n}$ \\
\hline+ & + & - & + & + & - \\
\hline
\end{tabular}

However, if we take into account the conditions on the coefficients, we see some open problems left, and different answers for $\bar{d}_{n}$ and $\bar{a}_{n}$ may be obtained. Following is the table of conditions on dominance coefficients and answers to the questions whether these conditions are sufficient for prediction (coefficients bounded from below are included for the sake of completeness).

\begin{tabular}{|c|c|c|c|c|c|c|}
\hline & $\mathbf{E} \bar{d}_{n}$ & $\bar{d}_{n}$ & $d_{n}$ & $\mathbf{E} \bar{a}_{n}$ & $\bar{a}_{n}$ & $a_{n}$ \\
\hline $\log c_{n}^{-1}=o(n)$ & + & $?$ & - & + & $?$ & - \\
\hline$\sum_{n=1}^{\infty} \frac{\log c_{n}^{-1}}{n^{2}}<\infty$ & + & + & - & + & + & - \\
\hline \hline$c_{n} \geq c>0$ & + & + & + & + & + & + \\
\hline
\end{tabular}

We know form Proposition 7 that the condition $c_{n} \geq c>0$ for convergence in $d_{n}$ can not be improved; thus the open problem left is to find whether $\log c_{n}^{-1}=o(n)$ is sufficient for prediction in $\bar{d}_{n}$ or at least in $\bar{a}_{n}$.

Another open problem is to find whether any conditions on dominance coefficients are necessary for prediction; so far we only have some sufficient conditions. On the one hand, the obtained results suggest that some form of dominance with 
decreasing coefficients may be necessary for prediction, at least in the sense of convergence of averages. On the other hand, the condition (1) is uniform over all sequences which probably is not necessary for prediction. As for prediction in the sense of almost sure convergence, perhaps more subtle behavior of the ratio $\frac{\mu\left(x_{1: n}\right)}{\rho\left(x_{1: n}\right)}$ should be analyzed, since dominance with decreasing coefficients is not sufficient for prediction in this sense.

\section{Preservation of the Predictive Ability under Summation with an Arbitrary Measure}

Now we turn to the question whether, given a measure $\rho$ that predicts a measure $\mu$ in some sense, the "contaminated" measure $(1-\varepsilon) \rho+\varepsilon \chi$ for some $0<\varepsilon<1$ also predicts $\mu$ in the same sense, where $\chi$ is an arbitrary measure. Since most considerations are independent of the choice of $\varepsilon$, in particularly the results in this section, we set $\varepsilon=\frac{1}{2}$ for simplicity. We define

Definition 8 (Contamination) By " $\rho$ contaminated with $\chi$ " we mean $\rho^{\prime}:=\frac{1}{2}(\rho+$ $\chi)$.

Positive results can be obtained for convergence in expected average KL divergence. The statement of the next proposition in a different form was mentioned in [RA06, Hut06]. Since the proof is simple we present it here for the sake of completeness; it is based on the same ideas as the proof of Theorem 5 .

Proposition $9(\mathbf{E} \overline{\boldsymbol{d}} \rightarrow \mathbf{0})$ Let $\mu$ and $\rho$ be two measures on $\mathcal{X}^{\infty}$ and suppose that $\rho$ predicts $\mu$ in expected average $K L$ divergence. Then so does the measure $\rho^{\prime}=\frac{1}{2}(\rho+\chi)$ where $\chi$ is any other measure on $\mathcal{X}^{\infty}$.

Proof.

$$
\begin{aligned}
0 \leq \mathbf{E} \bar{d}_{n}\left(\mu, \rho^{\prime}\right) & =\frac{1}{n} \mathbf{E} \sum_{t=1}^{n} \sum_{x_{t} \in \mathcal{X}} \mu\left(x_{t} \mid x_{<t}\right) \log \frac{\mu\left(x_{t} \mid x_{<t}\right)}{\rho^{\prime}\left(x_{t} \mid x_{<t}\right)}=\frac{1}{n} \mathbf{E} \log \frac{\mu\left(x_{1: n}\right)}{\rho^{\prime}\left(x_{1: n}\right)} \\
& =\frac{1}{n} \mathbf{E} \log \frac{\mu\left(x_{1: n}\right)}{\rho\left(x_{1: n}\right)} \frac{\rho\left(x_{1: n}\right)}{\rho^{\prime}\left(x_{1: n}\right)}=\mathbf{E} \bar{d}_{n}(\mu, \rho)+\frac{1}{n} \mathbf{E} \log \frac{\rho\left(x_{1: n}\right)}{\rho^{\prime}\left(x_{1: n}\right)},
\end{aligned}
$$

where the first term tends to 0 by assumption and the second term is bounded from above by $\frac{1}{n} \log 2 \rightarrow 0$. Since the sum is bounded from below by 0 we obtain the statement of the proposition.

Next we consider some negative results. An example of measures $\mu, \rho$ and $\chi$ such that $\rho$ predicts $\mu$ in absolute distance (or KL divergence) but $\frac{1}{2}(\rho+\chi)$ does not, can be constructed similarly to the example from [KL92] (of a measure $\rho$ which is a sum of distributions arbitrarily close to $\mu$ yet does not predict it). The idea is to take a measure $\chi$ that predicts $\mu$ much better than $\rho$ on almost all steps, but on some steps gives grossly wrong probabilities. 
Proposition $10(\boldsymbol{a} \nrightarrow \mathbf{0}$ and $\boldsymbol{d} \nrightarrow \mathbf{0})$ There exist measures $\mu, \rho$ and $\chi$ such that $\rho$ predicts $\mu$ in absolute distance (KL divergence) but $\frac{1}{2}(\rho+\chi)$ does not predict $\mu$ in absolute distance (KL divergence).

Proof. Let $\mu$ be concentrated on the sequence $11111 \ldots$ (that is $\mu\left(x_{n}=1\right)=1$ for any $n)$, and let $\rho\left(x_{n}=1\right)=\frac{n}{n+1}$ with probabilities independent on different trials. Clearly, $\rho$ predicts $\mu$ in both absolute distance and KL divergence. Let $\chi\left(x_{n}=1\right)=1$ for all $n$ except on the sequence $n=n_{k}=2^{2^{k}}=n_{k-1}^{2}, k \in \mathbb{N}$ on which $\chi\left(x_{n_{k}}=1\right)=$ $n_{k-1} / n_{k}=2^{-2^{k-1}}$. This implies that $\chi\left(1_{1: n_{k}}\right)=2 / n_{k}$ and $\chi\left(1_{1: n_{k}-1}\right)=\chi\left(1_{1: n_{k-1}}\right)=$ $2 / n_{k-1}=2 / \sqrt{n_{k}}$. It is now easy to see that $\frac{1}{2}(\rho+\chi)$ does not predict $\mu$, neither in absolute distance nor in KL divergence. Indeed for $n=n_{k}$ for some $k$ we have

$$
\frac{1}{2}(\rho+\chi)\left(x_{n}=1 \mid 1_{<n}\right)=\frac{\rho\left(1_{1: n}\right)+\chi\left(1_{1: n}\right)}{\rho\left(1_{<n}\right)+\chi\left(1_{<n}\right)} \leq \frac{1 /(n+1)+2 / n}{1 / n+2 / \sqrt{n}} \rightarrow 0 .
$$

For the (expected) average absolute distance the negative result also holds:

Proposition $11(\overline{\boldsymbol{a}} \nrightarrow \mathbf{0})$ There exist such measures $\mu, \rho$ and $\chi$ that $\rho$ predicts $\mu$ in average absolute distance but $\frac{1}{2}(\rho+\chi)$ does not predict $\mu$ in (expected) average absolute distance.

Proof. Let $\mu$ be Bernoulli $1 / 2$ distribution and let $\rho\left(x_{n}=1\right)=1 / 2$ for all $n$ (independently of each other) except for some sequence $n_{k}, k \in \mathbb{N}$ on which $\rho\left(x_{n_{k}}=1\right)=0$. Choose $n_{k}$ sparse enough for $\rho$ to predict $\mu$ in the average absolute distance. Let $\chi$ be Bernoulli 1/3. Observe that $\chi$ assigns non-zero probabilities to all finite sequences, whereas $\mu$-a.s. from some $n$ on $\rho\left(x_{1: n}\right)=0$. Hence $\frac{1}{2}(\rho+\chi)\left(x_{1: n}\right)=\frac{1}{2} \chi\left(x_{1: n}\right)$ and so $\frac{1}{2}(\rho+\chi)$ does not predict $\mu$.

Thus for the question of whether predictive ability is preserved when an arbitrary measure is added to the predictive measure, we have the following table of answers.

\begin{tabular}{|c|c|c|c|c|c|}
\hline $\mathbf{E} \bar{d}_{n}$ & $\bar{d}_{n}$ & $d_{n}$ & $\mathbf{E} \bar{a}_{n}$ & $\bar{a}_{n}$ & $a_{n}$ \\
\hline+ & $?$ & - & - & - & - \\
\hline
\end{tabular}

As it can be seen, there is one open question: whether this property is preserved under almost sure convergence of the average KL divergence.

It can be inferred from the example in Proposition 10 that contaminating a predicting measure $\rho$ with a measure $\chi$ spoils $\rho$ if $\chi$ is better than $\rho$ on almost every step. It thus can be conjectured that adding a measure can only spoil a predictor on sparse steps, not affecting the average. 
Conjecture $12(\boldsymbol{a} \rightarrow \mathbf{0}$ implies $\overline{\boldsymbol{a}} \rightarrow \mathbf{0})$ Suppose that a measure $\rho$ predicts a measure $\mu$ in absolute distance. Then for any measure $\chi$ the measure $\frac{1}{2}(\rho+\chi)$ predicts $\mu$ in average absolute distance.

As far as KL divergence is concerned we expect even a stronger conjecture to be true, since limited KL divergence does not allow a predicting measure to be (too close to) zero on any step.

Conjecture $13(\overline{\boldsymbol{d}} \rightarrow \mathbf{0})$ Suppose that a measure $\rho$ predicts a measure $\mu$ in average $K L$ divergence. Then for any measure $\chi$ the measure $\frac{1}{2}(\rho+\chi)$ predicts $\mu$ in average KL divergence.

\section{Miscellaneous}

Special cases of dominance with decreasing coefficients. In Section 3 we have shown that Laplace's measure $\rho_{L}$ for $\mathcal{X}=\{0,1\}$ dominates any Bernoulli i.i.d. measure with linearly decreasing coefficients. It can also be shown that a generalization of $\rho_{L}$ to a measure $\rho_{L}^{k}$ for predicting any measure with memory $k$, for a given $k$, dominates any such measure with polynomially decreasing coefficients (namely, $c_{n}^{-1}=\mathcal{O}\left(n^{|\mathcal{X}|^{k}}\right)$. The measure $\rho_{R}$ from [Rya88] for predicting any stationary measure was constructed as a sum of $\rho_{L}^{k}$ with positive weights: $\rho_{R}\left(x_{1 . . n}\right)=\sum_{k=1}^{\infty} w_{k} \rho_{L}^{k}\left(x_{1 . . n}\right)$. By construction, $\rho_{R}$ dominates any finite memory measure with polynomially decreasing coefficients. It is interesting to find whether $\rho_{R}$ (or any other measure which predicts all stationary measures) dominates every stationary measure with some subexponentially decreasing coefficients (or at least dominates non-uniformly). Clearly, this is a special case of the general open question - whether some form of dominance with decreasing coefficients is necessary for prediction.

Special questions of summation of a predictor with arbitrary measures. Although we know that adding a measure may spoil a predicting measure, it may be that carefully selecting which groups of measures to sum we can save all their predicting properties. One of the interesting cases is Zvonkin-Levin [ZL70] universal semi-computable measure ${ }^{2}$

$$
\xi\left(x_{1: n}\right)=\sum_{i \in \mathbb{N}} w_{i} \nu_{i}\left(x_{1: n}\right)
$$

where $\left(\nu_{i}\right), i \in \mathbb{N}$ is the class of all lower semi-computable semi-measures, and $w_{i}>0$. Since $\xi(A) \geq w_{i} \nu_{i}(A)$ for any measurable set $A$ ( $\xi$ dominates any $\nu_{i}$ with a constant $\left.w_{i}\right)$, it predicts all $\nu_{i}$ in the sense of convergence in total variation, in KL divergence and absolute distance. The question is what else does it predict,

\footnotetext{
${ }^{2}$ In fact $\xi$ is not a measure but only a semi-measure, but a semi-measure is sufficient for making predictions and it will not affect our arguments further.
} 
which other measures? Laplace's measure $\rho_{L}$ is computable, and hence is present in the sum (4). We know that $\rho_{L}$ predicts any Bernoulli i.i.d. measure, so we can ask whether $\xi$, being a sum of $\rho_{L}$ and some other measures, still predicts all Bernoulli measures. The predictor $\rho_{R}$ from Rya88 is computable and predicts all stationary measures in average KL divergence (and average absolute distance). Thus $\xi$ is a sum of $\rho_{R}$ and some other measure, and we can ask whether it still predicts all stationary measures. (In expected average KL divergence this follows from Proposition 9 as also pointed out in [Hut06].)

Conjecture 14 ( $a \rightarrow 0$ for i.i.d. and $\overline{\boldsymbol{d}} \rightarrow \mathbf{0}$ for stationary) For every i.i.d. measure $\nu$, the measure $\xi$ as defined in (4) predicts $\nu$ in absolute distance. For every stationary measure $\nu$, the measure $\xi$ predicts $\nu$ in average $K L$ divergence.

Proof idea. For the first question, consider any Bernoulli i.i.d. measure $\nu$. From Conjecture 12 and from the fact that $\rho_{L}$ predicts $\nu$ in absolute distance we conclude that $\xi$ predicts $\nu$ in average absolute distance. Since $\nu$ is Bernoulli i.i.d. measure, that is, probabilities on all steps are equal and independent of each other, from any measure $\theta$ that predicts $\nu$ in average absolute distance we can make a measure $\theta^{\prime}$ which predicts $\nu$ in absolute distance as follows $\theta^{\prime}\left(x_{n}=x \mid x_{<n}\right)=\frac{2}{n} \sum_{t=n / 2}^{n} \theta\left(x_{t}=\right.$ $\left.x \mid x_{<n}\right)$. Moreover, the convergence speed of $\theta^{\prime}$ to $\nu$ will be the same as that of $\theta$ to $\nu$. But if $\xi$ is semi-computable then so is $\xi^{\prime}$, so that $\xi^{\prime}$ is present in the sum (4). Since $\xi$ is not a better predictor than $\xi^{\prime}$, adding $\xi$ to $\xi^{\prime}$ can not spoil the latter.

If $\nu$ is a stationary measure, then we known from Proposition 9 and that $\rho_{R}$ is computable that $\xi$ predicts $\nu$ in expected average KL divergence (absolute distance). Conjecture 13 would also imply that $\xi$ predicts $\nu$ in average KL divergence, and average absolute distance.

Other measures of divergence. The last question we discuss is criteria of prediction other than introduced in Section 2. Apart form the measures of divergence of probability measures that we considered we mention also the following:

(s) squared distance $s_{n}\left(\mu, \rho \mid x_{<n}\right)=\sum_{x \in \mathcal{X}}\left(\mu\left(x_{n}=x \mid x_{<n}\right)-\rho\left(x_{n}=x \mid x_{<n}\right)^{2}\right.$,

(h) Hellinger distance $h_{n}\left(\mu, \rho \mid x_{<n}\right)=\sum_{x \in \mathcal{X}}\left(\sqrt{\mu\left(x_{n}=x \mid x_{<n}\right)}-\sqrt{\rho\left(x_{n}=x \mid x_{<n}\right.}\right)^{2}$,

the average squared distance $\bar{s}_{n}$ and the average Hellinger distance $\bar{h}_{n}$ are introduced analogously to $\bar{a}_{n}$ and $\bar{d}_{n}$. It is easy to check that all negative results obtained hold with respect to $s_{n}$ and $h_{n}$ as well. Positive results for $s_{n}$ and $h_{n}$ follow from corresponding positive results for KL divergence $d_{n}$ and inequalities $s_{n}(\mu, \rho) \leq d_{n}(\mu, \rho)$ and $h_{n}(\mu, \rho) \leq d_{n}(\mu, \rho)$, see e.g. [Hut05, Lem.3.11]. Expected absolute convergence $\mathbf{E} a_{n} \rightarrow 0$ (also called convergence in the mean) and expected KL convergence $\mathbf{E} d_{n} \rightarrow 0$ may also be considered. 


\section{Outlook and Conclusion}

In the present work we formulated and started to address the question for which classes of measures sequence prediction is possible. Towards this aim we defined the notion of dominance with decreasing coefficients (a condition on local absolute continuity) and found some forms of it which are sufficient for prediction. We have also addressed the question which forms of predictive ability are preserved under "contamination" of a predictor by an arbitrary measure. Besides some more concrete open problems posed in the corresponding sections, a program for answering the general questions formulated can be outlined as follows: We would like to find some conditions on dominance with decreasing coefficients which are necessary and sufficient for prediction; for those notions of prediction ability for which this is not possible, more subtle behavior of the ratio $\frac{\mu\left(x_{1: n}\right)}{\rho\left(x_{1: n}\right)}$ should be analyzed to obtain conditions both necessary and sufficient for prediction. This should give rise to an abstract characterization of classes of measures for which a measure satisfying such conditions for all measures in the class exists; that is, to a description of classes of measures for which prediction is possible. It is expected that such characterization will naturally lead to a construction of a predictor as well - perhaps in form of a Bayesian integral. The latter conjecture also encourages studying the question of "contamination" of a predictor with arbitrary measures. The next step will be to extend this approach to the task of active learning [Hut05, RH06].

\section{References}

[BD62] D. Blackwell and L. Dubins. Merging of opinions with increasing information. Annals of Mathematical Statistics, 33:882-887, 1962.

[Hut05] M. Hutter. Universal Artificial Intelligence: Sequential Decisions based on Algorithmic Probability. Springer, Berlin, 2005. 300 pages, http://www.idsia.ch/ marcus/ai/uaibook.htm.

[Hut06] M. Hutter. On the foundations of universal sequence prediction. In Proc. 3rd Annual Conference on Theory and Applications of Models of Computation (TAMC'06), volume 3959 of $L N C S$, pages 408-420. Springer, 2006.

[JKS99] M. Jackson, E. Kalai, and R. Smorodinsky. Bayesian representation of stochastic processes under learning: de Finetti revisited. Econometrica, 67(4):875-794, 1999.

[KL92] E. Kalai and E. Lehrer. Bayesian forecasting. Discussion paper 998, 1992.

[RA06] B. Ryabko and J. Astola. Universal codes as a basis for time series testing. Statistical Methodology, To appear, available online, 2006.

[RH06] D. Ryabko and M. Hutter. Asymptotic learnability of reinforcement problems with arbitrary dependence. Technical Report IDSIA-09-06, 2006. http://arxiv.org/abs/cs.LG/0603110. 
[Rya88] B. Ryabko. Prediction of random sequences and universal coding. Problems of Information Transmission, 24:87-96, 1988.

[Shi96] A. N. Shiryaev. Probability. Springer, 1996.

[Sol64] R. J. Solomonoff. A formal theory of inductive inference: Part 1 and 2. Inform. Control, 7:1-22, 224-254, 1964.

[Sol78] R. J. Solomonoff. Complexity-based induction systems: comparisons and convergence theorems. IEEE Trans. Information Theory, IT-24:422-432, 1978.

[ZL70] A. K. Zvonkin and L. A. Levin. The complexity of finite objects and the development of the concepts of information and randomness by means of the theory of algorithms. Russian Mathematical Surveys, 25(6):83-124, 1970. 